\title{
The role of omalizumab or rhuMAb-E25 in the treatment of allergic rhinitis
}

\section{To the Editor:}

I read with interest the recent article by HANF et al. [1], demonstrating the significant inhibition of both allergen challenge-induced nasal symptoms and the increase of human serum albumin in nasal lavage fluid after allergen challenge, in addition to the significant reduction in tumour necrosis factor- $\alpha$ levels in basal nasal lavage fluid, following administration of omalizumab.

However, it is difficult to gauge from the study, the exact degree of disease severity in the study population, as no data were given with regards to the patients' usual therapy for allergic rhinitis, be it topical or oral, and hence no assessment of correlation between treatment and symptoms can be made. In addition, the characterisation of the study population was inadequate, as to whether patients suffered from seasonal or perennial allergic rhinitis, as no detailed data with regards to each individual's aeroallergen sensitisation were presented. Moreover, one has to be wary of the potential confounding effects of conducting the study during the pollen season, especially in patients who were sensitised to birch and grass pollen.

Although the study did shed some light on the potential for the use of omalizumab in allergic rhinitis, the exact indications for its use remain unclear. It is a pity that patients were not better categorised or characterised, as one could have perhaps argued a case for the role of omalizumab as a second- or third-line agent following failure with conventional therapy, such as corticosteroids, histamine $\mathrm{H}_{1}$-receptor antagonists and leukotriene $\mathrm{CysLT}_{1}$-receptor antagonists. Furthermore, the fact that the mode of delivery of omalizumab is through subcutaneous administration must not be ignored, as this will disadvantage it against other established first-line topical or oral therapy for allergic rhinitis.

\section{D.K.C. Lee}

Dept of Respiratory Medicine, Ipswich Hospital, Ipswich, UK.

\section{References}

1. Hanf G, Noga $\mathrm{O}, \mathrm{O}^{\prime}$ Connor A, et al. Omalizumab inhibits allergen challenge-induced nasal response. Eur Respir J 2004; 23: 414418 .

\section{From the authors:}

In our recent article, we demonstrated the inhibitory effect of subcutaneously administered monoclonal anti-immunoglobulin-E antibody, omalizumab, on nasal responses to allergen challenge in patients with allergic rhinitis [1]. Omalizumabtreated patients showed reduced nasal symptoms, an inhibition of the increase of human serum albumin in the nasal lavage fluid after allergen challenge and a decrease in tumour necrosis factor- $\alpha$ in basal nasal lavage fluids.

D.K.C. Lee mentioned in his Letter to the Editor, that no exact degree of disease severity and no data with regards to the usual therapy for allergic rhinitis were given in the article. Moreover, D.K.C. Lee missed an exact indication for the use of omalizumab and an estimation of the role of omalizumab in the therapy of allergic rhinitis compared to the conventional therapy. Furthermore, D.K.C. Lee missed detailed data with regards to each individual's aeroallergen and mentioned a possible influence of the pollen season on the results of our study. Finally, D.K.C. Lee stresses the subcutaneous way of application of omalizumab, as this will be a disadvantage against the established therapies for allergic rhinitis.

The target of our study was the effect of omalizumab on the nasal symptoms and the inflammatory markers in nasal lavage fluid before and after allergen challenge of patients with allergic rhinitis. The degree of disease severity and the usual therapy were not a target of our study, moreover the patients had no symptoms at the time-points of allergen challenge. However, we think the influence of severity and usual therapy would be very interesting for further studies.

As our study was a model of allergen provocation, of course it was not possible to get exact results for the indication for omalizumab in the therapy of allergic rhinitis. There are other studies which have investigated the effect of omalizumab on allergic rhinitis during the allergy season [2]. Further studies like these may have the possibility to analyse the rank of omalizumab in the therapy of allergic rhinitis. At this time, omlizumab is approved for the additional treatment of severe asthma. From our point of view, omalizumab has its advantage in the treatment of multiple allergic diseases, such as allergic asthma and allergic rhinitis simultaneously.

As mentioned above, all patients who participated in the study were symptom-free at the time-points of allergenchallenge. This includes the seasonal und perenial allergens. Consequently, we had no influence of seasonal effects on the results of our study.

The subcutaneous application of omalizumab was no problem for our patients. Yet we agree with D.K.C. Lee, that we would prefer a different way of administration or a longer period between the injections. However, the route of administration is defined by the pharmacokinetics and pharmacodynamics of the compound.

Over all, the interest of our studies of the effect of omalizumab was mainly the influence on inflammatory processes. As in the recently published article concerning allergic asthma [3], we focused on the description of the effect of omalizumab on allergic inflammation to get a more detailed insight into the reasons of the clinical effect of omalizumab.

\section{G. Hanf, O. Noga}

Allergy and Asthma Clinic, Charité, Humboldt University Berlin, Germany.

\section{References}

1. Hanf $\mathrm{G}$, Noga $\mathrm{O}, \mathrm{O}^{\prime}$ Connor A, et al. Omalizumab inhibits allergen challenge-induced nasal response. Eur Respir J 2004; 23: $414-418$. 